\title{
Accuracy of Thermal Imaging Camera in Identification of Perforators
}

\author{
Muhammad Jibran Rabbani, Asma Ilyas, Ammara Rabbani, Zain ul Abidin and Moazzam Nazeer Tarar \\ Jinnah Burn and Reconstructive Surgery Centre, AIMC, Lahore, Pakistan
}

\begin{abstract}
Objective: To determine the diagnostic accuracy of thermal imaging camera in the identification of perforators using peroperative visual inspection as gold standard.

Study Design: Descriptive study.

Place of Study: Jinnah Burn and Reconstructive Surgery Centre / Allama Iqbal Medical College, Lahore, from October 2017 to September 2018.

Methodology: Patients undergoing pedicled or free perforator flap for reconstructive surgery of upper and lower limb, abdomen, groin, and head and neck region, where soft tissue defect was caused by trauma (road traffic accidents and electric burns), were included in this study. All patients underwent the same treatment protocol of identification of perforator location with Flir One camera preoperatively. Later, it was confirmed peroperatively by visual inspection. Diagnostic accuracy was calculated in terms of sensitivity, specificity, positive predictive value, and negative predictive value.

Results: Diagnostic accuracy in identification of perforators in pedicled $(n=154)$ and free flaps $(n=30)$ by thermal imaging camera was calculated as sensitivity of $86.2 \%$, specificity of $80.0 \%$ with the positive and negative predictive values of $98.7 \%$, and $25.0 \%$, respectively.

Conclusion: Flir One, a smartphone-compatible miniature thermal imaging camera, is useful for the detection of perforators and is highly sensitive and specific.
\end{abstract}

Key Words: Flir One, Perforators, Thermal imaging, Thermal imaging camera.

How to cite this article: Rabbani MJ, Ilyas A, Rabbani A, Abidin ZU, Tarar MN. Accuracy of Thermal Imaging Camera in Identification of Perforators. J Coll Physicians Surg Pak 2020; 30(05):512-515. DOI: https://doi.org/10.29271/jcpsp.2020.05.512.

\section{INTRODUCTION}

Perforatorflaps, pedicled or free, are commonly used for the reconstruction of soft tissue defects. ${ }^{1}$ Successful surgery requires accurate knowledge of underlying vascular anatomy and the exact location of perforators. Unfortunately, often dissection is challenging due to variability of perforators course and their location. ${ }^{2}$ Various landmark studies have been done to find the blood supply from a source artery to the skin on cadavers and humans as well. ${ }^{3}$ More recently, hand-held Doppler, Duplexultrasound, computed tomography, and magnetic resonance angiography and thermography methods have been used preoperatively to detect the location of the perforating vessels. ${ }^{4}$ There are marked disadvantages of each method. The hand-held Doppler ultrasound is time-consuming and may have false positive or negative results. ${ }^{5}$ Subjective findings, depending on the expertise of radiologists, are appreciated on duplex ultrasound, computed tomography, and magnetic resonance angiography. In addition, they expose patients to the risk of intravenous contrast and are, therefore, less accessible and more expensive..$^{2-4}$

Correspondence to: Dr. Muhammad Jibran Rabbani, Jinnah

Burn and Reconstructive Surgery Centre, AIMC, Lahore,

Pakistan

E-mail: jibranrabbani@hotmail.com

Received: February 06, 2020; Revised: May 18, 2020;

Accepted: May 27, 2020

DOI: https://doi.org/10.29271/jcpsp.2020.05.512
Infrared radiations are detected on thermal imaging devices that produce thermogram, and thus, cutaneous blood flow can beinterpreted as a surrogate marker. $^{6}$

A smartphone compatible small thermal imaging camera, The Flir One (Flir Systems, Inc., Wilsonville, Ore.) currently costs about $\$ 200$. It uses an infrared sensor with a long-wavelength (8 to $14 \mu \mathrm{m}$ ) that has a working temperature range of $0^{\circ}$ to $100^{\circ} \mathrm{C}$ but, because of its compact nature, provides a lower resolution image, with a narrower temperature detection range than the more expensive thermal imaging cameras. It requires minimal training and is a simple "point-and-shoot" technology. ${ }^{7,8}$ In one study, ${ }^{8}$ the accuracy of thermal imaging camera in the identification of perforators was $100 \%$, and $97.5 \%$ in another study ${ }^{2}$. Both studies used visual method as the gold standard. In another study, Weim et al. showed a $95.6 \%$ sensitivity for perforator detection. ${ }^{9}$ In another study, the sensitivity, positive predictive value, and accuracy of infrared thermography is $95.05 \%, 80.67 \%$, and $77.41 \%$, respectively. ${ }^{10} \mathrm{~A}$ different study showed a sensitivity of $33 \%{ }^{11}$, a specificity of $75 \%,{ }^{12}$ and prevalence of $57 \%{ }^{13}$ in detecting perforators.

A non-invasive, non-touch, reliable technique that can be used routinely by the plastic surgeon for theidentification and preservation of perforators, is vital for the successful planning of reconstructive surgery. The present study aimed to determine the diagnostic accuracy of the thermal imaging camera in the identification of perforators. 
Table I: Diagnostic accuracy of thermal imaging.

\begin{tabular}{|c|c|c|c|c|}
\hline \multirow{2}{*}{ Thermal scan } & \multicolumn{2}{|c|}{ Visual inspection } & \multirow{2}{*}{ Total } & \multirow{2}{*}{$\begin{array}{c}\text { Fisher's Exact } \\
\text { p-value }\end{array}$} \\
\hline & Yes & No & & \\
\hline \multirow{3}{*}{ Yes } & 150 & 2 & 152 & \multirow{9}{*}{$\begin{array}{c}x^{2}=28.852 \\
p<0.001\end{array}$} \\
\hline & $98.7 \%$ & $1.3 \%$ & $100.0 \%$ & \\
\hline & $86.2 \%$ & $20.0 \%$ & $82.6 \%$ & \\
\hline \multirow{3}{*}{ No } & 24 & 8 & 32 & \\
\hline & $75.0 \%$ & $25.0 \%$ & $100.0 \%$ & \\
\hline & $13.8 \%$ & $80.0 \%$ & $17.4 \%$ & \\
\hline \multirow{3}{*}{ Total } & 174 & 10 & 184 & \\
\hline & $94.6 \%$ & $5.4 \%$ & $100.0 \%$ & \\
\hline & $100.0 \%$ & $100.0 \%$ & $100.0 \%$ & \\
\hline
\end{tabular}

METHODOLOGY

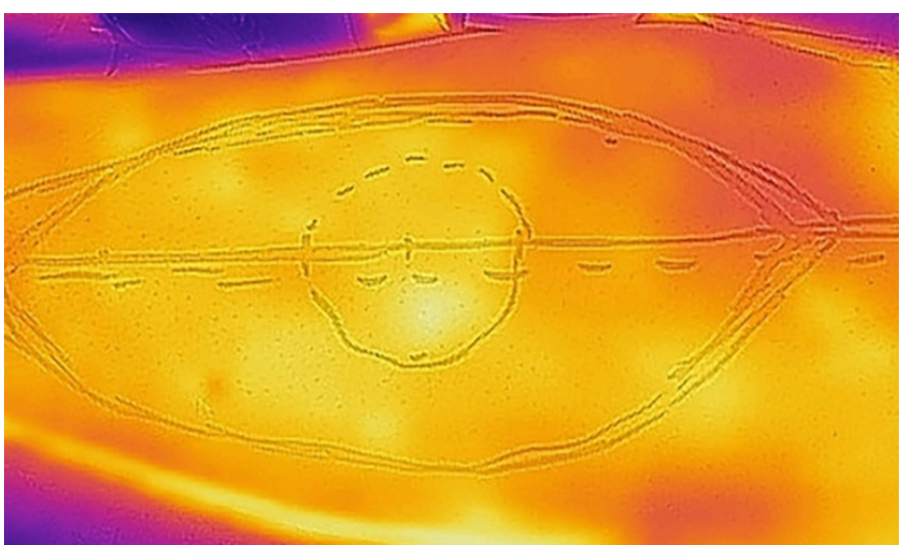

a
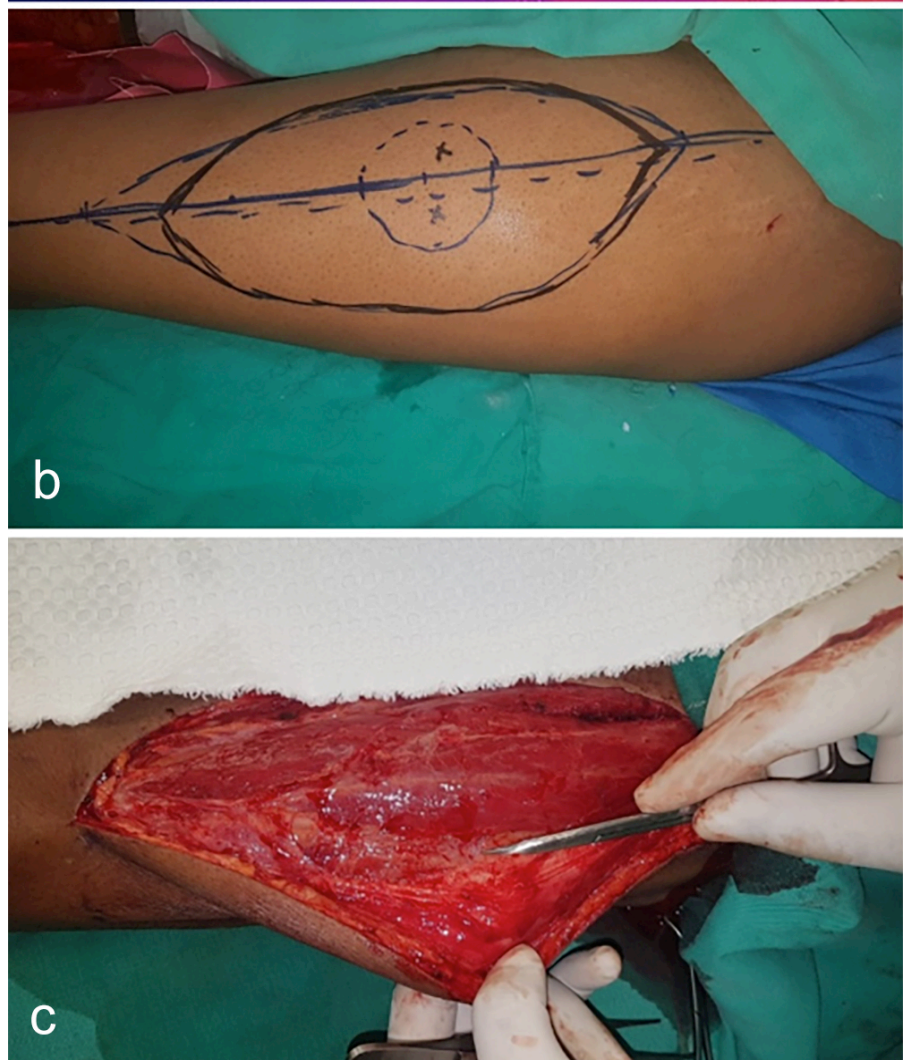

Figure 1: Perforator Identification; (a) Pre-op visualisation of perforators (hot spots) on thermal imaging; (b) Pre-op marking of perforators on leg with handheld doppler; (c) Intra-operative identification and confirmation of perforators.
A total of 184 patients were enrolled from Jinnah Burn and Reconstructive Surgery Centre/Allama Iqbal Medical College, Lahore, from October 2017 to September 2018. All patients undergoing pedicled or free perforator flap reconstructive surgery, aged 16 to 38 years of either gender, were included. Patients with hyperthermia $\left(>99.5^{\circ} \mathrm{F}\right)$ or hypothermia $\left(<98.6^{\circ} \mathrm{F}\right)$ were in the exclusion list, as this could affect perforator detection and thermo-gram. ${ }^{14}$ Informed written consent was taken from each patient. The area to be examined was left exposed to room temperature for at least two minutes before the procedure. Identification of perforator location with Flir One (a smartphone compatible thermal imaging camera) was done. Perforators were confirmed by peroperative visual inspection as a gold standard to evaluate the diagnostic accuracy of the thermal imaging camera (Figure 1). Effect modifiers like age and gender were addressed through the stratification of data.

Diagnostic accuracy was calculated using a $2 \times 2$ table with peroperative visual inspection as a gold standard (Table I). True positive (TP - perforators detected on TI and visual inspection as well), true negative (TN - perforators not detected on TI and visual inspection as well), false positive (FP - perforators detected on $\mathrm{TI}$ but not on visual inspection) and false negative (FN - perforators not detected on TI but present on visual inspection) were noted. Accuracy was calculated in terms of sensitivity (ability of TI in the detection of perforators). TP / (TP + FN) $x 100$, specificity (ability of TI to identify the absence of perforators). TN / (TN + FP) x 100, positive predictive value (PPV - proportion of perforators presence in all positive cases). TP / (TP + FP) x 100 and negative predictive value (NPV proportion of no perforators among all negative cases) TN / $(\mathrm{TN}+\mathrm{FN}) \times 100$. The collected data was entered and analysed using SPSS version 20. Mean and the standard deviation were calculated for numerical variables like age. Frequencies and percentages were recorded for categorical variables. Diagnostic accuracy, sensitivity, specificity, PPV, and NPV were calculated using cross-tabulation between thermal imaging and visual inspection. Data was stratified for age, gender, and location of the flap. Post-stratification diagnostic accuracy calculated and Fisher's exact test applied with p-value $\leq 0.05$ were considered as significant.

\section{RESULTS}

The age range of selected 184 patients was $16-38$ years, mean 
was $23.34+6.06$ years. There were $125(67.93 \%)$ males, and $59(32.07 \%)$ females. The total number of flaps harvested from the upper limb was 30 (16.30\%), lower limb was $41(22.28 \%)$, abdomen 69 (37.50\%), and groin was 44 (23.91\%). One hundred and fifty-four flaps were pedicled (83.7\%), and 30 (16.3\%) were free flaps (Figure 1). The diagnostic accuracy of thermal imaging scan was found to be $85.9 \%$ with a sensitivity of $86.2 \%$, a specificity of $80 \%$, a positive predictive value of $98.7 \%$, and a negative predicted value of $25 \%$.

\section{DISCUSSION}

The objective of this study was to assess the clinical application of thermal imaging camera in the domain of plastic surgery. Identification of suitable perforation is a crucial step in planning and designing a flap for reconstruction. At present, available imaging techniques employed for preoperative mapping are hand-held doppler, duplex ultrasound, CTA (computed tomography angiogram), and MRA (magnetic resonance angiography). ${ }^{11-13}$ Doppler ultrasound yields $91.3 \%$ sensitivity ${ }^{10}$ and it requires long examination time and the need for an experienced operator who possesses detailed knowledge of perforator anatomy. ${ }^{15}$ CTA, MRA, and digital subtraction angiography (DSA) can be used to map the blood vessels and perforator accurately, but these methods are operator-dependent, expensive and are not available easily for intra-operative and postoperative monitoring. Furthermore, they expose patients to high radiation. Also, there is a risk of contrast dye-induced nephrotoxicity and extravasation injury. ${ }^{10}$ There is a need for a bedside device that is inexpensive, non-invasive, ideally contactless with high accuracy and sensitivity and has no or less adverse effects. Thermal imaging photography fulfills all the above and needs a simple procedure that does not require any expertise and can easily be performed by the operating surgeon in the preoperative clinic visit in a short period.

The authors assessed the accuracy of thermal imaging in the identification of perforators by verifying their location intraoperatively. Its sensitivity was found to be $86.2 \%$, and specificity was $80 \%$, with an accuracy of $85.9 \%$, which is quite comparable with the diagnostic accuracy of $95.6 \%{ }^{9}$ and $77.4 \%{ }^{10}$ in different studies. Intraoperative findings of perforators in different age groups were also compared in this study. A $91.2 \%$ accuracy in the age group below 30 years and $84.4 \%$ accuracy in above 30 years age group with a significant $p$ value of 0.001 was found. Near-identical results in both genders with accuracy of $86.9 \%$ and $88.1 \%$ in males and females, respectively were identified. While comparing thermal imaging with intraoperative visualisation of perforators, out of 184 , true positives were 150 (81.5\%), and 8 (4.3\%) were true negatives, which gives the high concordance. False positives were 2 (1.1\%), and false negatives were 24 (13.0\%). Pereira et al. found that smartphone-compatible thermal imaging cameras can detect cutaneous perforators with a sensitivity of $100 \%$ and specificity of $98 \%$, while taking CT angiography as a gold standard. ${ }^{16}$ The results of Pereira's study are in concordance with the present study. These results were a true reflection of thermal imaging's high accuracy.
Thermal imaging is a non-touch technique for vascular mapping that does not require exposure to contrast or radiation. Although prone to background thermal interference and artefact, if the skin is allowed a short period of acclimatisation to room temperature, perforator hotspots can readily be identified to aid localisation and confirmation with hand-held doppler ultrasound. Now, that real-time thermal imaging is widely available, it should be used in conjunction with existing technologies to provide additional clinical information that can be used to aid the assessment, execution, and postoperative monitoring for tissue transfer. It is easy to carry this small device that is a quick tool for screening and mapping. Although the resolution of the Flir One is lower than that of more expensive thermal imaging cameras, it provides a low cost alternative that can be applied to other domains of plastic surgery.

Authors of the present study find this device very useful, which uses the thermal image to locate the perforator accurately in relation to standard anatomical landmarks and thus help the surgeons making and executing a reconstruction plan reliably. Thermal imaging camera offers advantages of its compact structure, portability, ease of use while providing real-time images in detection in perforators. Consideration should be given to its adjunct use with other available perforator detection techniques. Very useful, yet it has certain limitations. A thermal imaging device only identifies the cutaneous part of perforator and cannot map the course of the vessel, which may easily be identified on more advanced technologies like CTA or MRA. Its best clinical use needs to be defined with further studies.

\section{CONCLUSION}

All the dominant perforators, identified by thermal scans, were confirmed at surgery. Thermographic imaging device has high sensitivity and specificity. This makes the said device very useful in the identification and mapping of perforators in free or pedicled perforator flap reconstruction, which saves operating time and thus decreases complication rate.

\section{CONFLICT OF INTEREST:}

The authors declared no conflict of interest, especially regarding the use of a medical device.

\section{ETHICAL APPROVAL:}

Ethical approval was taken from Ethical Committee of Jinnah Burn and Reconstructive Surgery Centre, Lahore prior to the initiation of the research work.

\section{PATIENTS' CONSENT:}

Informed consents were taken from all the patients to participate and publish the results obtained from the study.

\section{AUTHORS' CONTRIBUTION:}

MJR: Acquired data, data analysis, results preparation, write up, review, manuscript revision

Al, AR, AZ: Acquired data, results analysis, results preparation, manuscript review

MNT: Overall guidance, manuscript review, proof reading. 


\section{REFERENCES}

1. Geddes CR, Morris SF, Neligan PC. Perforator flaps: Evolution, classification, and applications. Ann Plast Surg 2003; 50:90-9.

2. Sheena Y, Jennison T, Hardwicke JT, Titley OG. Detection of perforators using thermal imaging. Plast Reconstr Surg 2013; 132:1603-10.

3. Salmon M, Taylor GI, Tempest MN. Arteries of the skin. Churchill Livingstone 1988.

4. Mathes DW, Neligan PC. Preoperative imaging techniques for perforator selection in abdomen-based microsurgical breast reconstruction. Clin Plast Surg 2010; 37:581-91.

5. Stekelenburg CM, Sonneveld PM, Bouman MB, van der Wal $\mathrm{MB}, \mathrm{Knol} \mathrm{DL}$, et al. The hand-held doppler device for the detection of perforators in reconstructive surgery: What you hear is not always what you get. Burns 2014; 40:1702-6.

6. Paul SP. Using a thermal imaging camera to locate perforators on the lower limb. Arch Plast Surg 2017; 44:243.

7. FLIR O. Veterin. Breda, NZ: FLIR Commercial Systems BV; cited 2017.

8. Hardwicke JT, Osmani O, Skillman JM. Detection of perforators using smartphone thermal imaging. Plast Reconstr Surg 2016; 137:39-41.

9. Weum S, Mercer JB, de Weerd L. Evaluation of dynamic infrared thermography as an alternative to $\mathrm{CT}$ angiography for perforator mapping in breast reconstruction: A clinical study.
BMC Med Imaging 2016; 16:43.

10. Muntean MV, Strilciuc S, Ardelean F, Pestean C, Lacatus R, Badea $A F$, et al. Using dynamic infrared thermography to optimise color doppler ultrasound mapping of cutaneous perforators. Med Ultrason 2015; 17:503-8.

11. Just M, Chalopin C, Unger M, Halama D, Neumuth $T$, Dietz $A$, et al. Monitoring of microvascular-free flaps following oropharyngeal reconstruction using infrared thermography: First clinical experiences. Eur Arch Oto-Rhino-Laryngol 2016; 273:2659-67.

12. Miland $\AA \mathrm{O}$, de Weerd L, Mercer JB. Intraoperative use of dynamic infrared thermography and indocyanine green fluorescence video angiography to predict partial skin flap loss. Eur J Plast Surg 2008; 30:269-76.

13. Bhandari $P$, Bath $A$, Sadhotra $L$, Singh $M$, Mukherjee $M$. Management of soft tissue defects of the ankle and foot. Med J Armed Forces India 2005; 61:253-5.

14. Vorvick L, Zieve D. ADAM editorial team (2018) Body temperature norms: MedlinePlus Medical Encyclopedia. Medlineplus. gov.

15. Taylor GI, Caddy CM, Watterson PA, Crock JG. The venous territories (venosomes) of the human body: Experimental study and clinical implications. Plast Reconstr Surg 1990; 86:185-213.

16. Pereira N, Valenzuela D, Mangelsdorff G, Kufeke M, Roa R. Detection of perforators for free flap planning using smart-phone thermal imaging: A concordance study with computed tomographic angiography in 120 perforators. Plast Reconstr Surg 2018; 141:787-92. 